\title{
Location Identification and Vehicle Tracking using VANET ( VETRAC )
}

\author{
Arunkumar Thangavelu ${ }^{1}$, K. Bhuvaneswari ${ }^{2}$, K. Kumar ${ }^{1}$, K. SenthilKumar ${ }^{1}$ and S.N. Sivanandam ${ }^{3}$
}

\begin{abstract}
Large cities with fleet of vehicles require a system to determine location of movement of passenger vehicles at a given time. Vehicle tracking systems can be used in theft prevention, retrieval of lost vehicles, providing trafficoriented services on lanes. The Vehicle tracking systems VETRAC enables vehicle drivers or any third party to track the location of any moving vehicle. Most modern vehicle tracking systems use GPS[7] modules which is costly in usage and implementation. Many systems also combine a communications component such as cellular or satellite transmitters to communicate the vehicle's location to a remote user. VETRAC uses WiFi IEEE $802.11 \mathrm{~b} / \mathrm{g}$ for easy and accurate location of the vehicle, which provides effective and simple communication. Vehicle information can be viewed on electronic maps using the Internet or specialized software. We have designed and developed an Intelligent Vehicle Navigation System, which identify an optimally minimal path for navigation with minimal traffic intensity using WiFi. The system can also be used as a city guide to locate and identify landmarks in a new city.
\end{abstract}

\section{INTRODUCTION}

An automotive navigation system[2],[12] is radio frequency based wirless-mobile navigation system designed to guide vehicles in its location using digital map[4] display. Using the $\mathrm{road} / \mathrm{path}$ database, system can give multiple directions to other locations to reach destination.

Vehicle tracking systems are electronic devices installed in vehicles to enable vehicle owners or third parties to track the location of a vehicle. Most modern vehicle tracking systems now use GPS [7] modules to allow for easy and accurate location of the vehicle. Many systems also combine a communications component such as cellular or satellite transmitters to communicate the vehicle's location to a remote user. Vehicle information can be viewed on electronic maps using the Internet or specialized software. The disadvantage of navigation system function by GPS is it cannot locate a vehicle which is inside big campuses like universities, airports, and tunnel [11]. The WiFi (Wireless Local Area Network) provides the navigation, tracking function in the big campuses area like universities, airports.

1 School of Computing Sciences, Vellore Institute of Technology, Vellore

2 Department of MCA, SNS College of Technology, Coimbatore

3 Department of Computer Science \& Engg, PSG College of Technology, Coimbatore
The goal is to create a system whereby vehicles can exchange information about traffic conditions in an effort to provide drivers with an early warning of traffic hazards and congestion. For example, when an accident occurs, emergency responders could provide information about road or lane closures and the estimated time of re-openings to oncoming traffic. The oncoming traffic could then carry the information to vehicles that have not yet reached the traffic slowdown. The navigation software in these approaching vehicles could then make an informed decision about whether or not to suggest an alternate route.

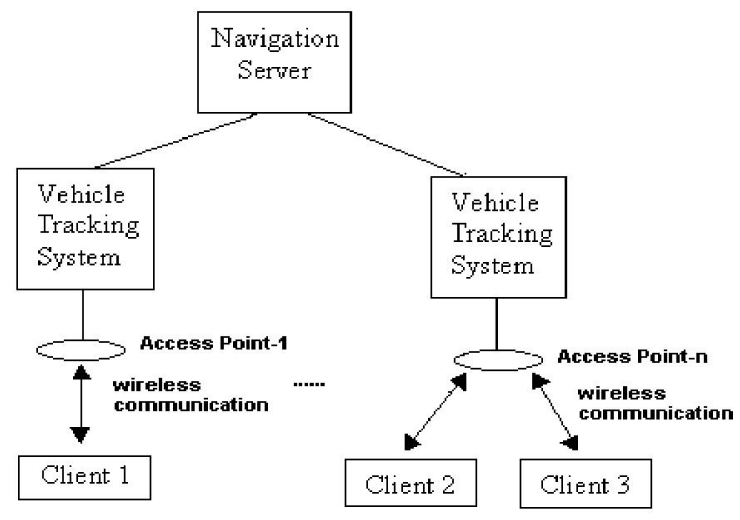

Fig. 1. Mobile Vehicle Tracking System

\section{A. AIM-VETRAC}

The aim of the project "VETRAC" is to make navigation system in vehicle using $\mathrm{WiFi}$ as the inter-connection networks. The carriers move around the location or hotspot where multiple WiFi access points are setup. The carriers gather information about current location, destination client's location and landmarks, also know about traffic information through streaming traffic video information. Each request from various clients, are serviced by WiFi enabled devices, which are established in the campus.

\section{B. MOTIVATION-VETRAC}

The motivation of this project "VETRAC" is

1. To minimize and eliminate congestion based traffic path during transit by mapping the vehicle to traffic in multiple paths.

2. To provide a safe and intelligent solution of identifying optimal paths to the vehicle driver.

3. To provide traffic video information this is useful for driver to know particular junction during traffic congestion. 
4. To identify the landmark to the vehicle driver or to a new entrant in the city.

5. To enable continuous communication (conference) with other vehicle through wire-free networks in non lineof-sight.

\section{BACKGROUND AND OVERVIEW}

Our goal is to create a system VETRAC where mobile vehicles can exchange information about traffic conditions in an effort to provide drivers with an early warning of traffic hazards and congestion. The system also considers emergency situations when an accident occurs, emergency responders could provide information about road or lane closures and the estimated time of re-openings to oncoming traffic. The oncoming traffic could then carry the information to vehicles that have not yet reached the traffic slowdown. The navigation software in these approaching vehicles could then make an informed decision about whether or not to suggest an alternate route. VETRAC is also provides information about a location for new entrants into city.

Achieving this goal requires research into forwarding information efficiently from providers (vehicles that detect the traffic event) through carriers (WiFi access-points installed at various points on lanes) to consumers (vehicles that need such information). The consumers will likely be hundreds of meters behind the providers, and therefore will be out of direct radio range (i.e., the providers and consumers are not in the same neighborhood and thus are not neighbors).

The vehicles form an ad-hoc network, meaning that there is no pre-arranged network infrastructure and that the vehicles are not required to have access to the public Internet for communication. In addition, these vehicles are on mobility, so a vehicular mobile ad-hoc network (VANET) is created. In particular, VANETs that consist of vehicles as nodes, face challenges that are far different than regular MANETs. These challenges include high-speed mobility, constraints on where vehicles can move because of roadways, unpredictable driver behavior and variable traffic behavior[2]

Some proposed inter-vehicular communication systems [4],[7] rely on the presence of static, outside infrastructure for connectivity or event notification (e.g., fixed info-stations that can monitor traffic and provide information to passing vehicles). In contrast, the techniques we develop can be applied to any set of vehicles that have a network device capable of sending and receiving messages. Other work [1],[3],[6] has considered passing information only through vehicles traveling in the same direction, rather than taking advantage of traffic in oncoming lanes.

\section{A. VANET}

Most inter-vehicular communication systems [4],[7] rely on the presence of static, outside infrastructure for connectivity or event notification (e.g., fixed info-stations that can monitor traffic and provide information to passing vehicles). In contrast, the techniques we develop can be applied to any set of vehicles that have a network device capable of sending and receiving messages. Other work [1], [3], [6] has considered passing information only through vehicles traveling in the same direction, rather than taking advantage of traffic in oncoming lanes.

Achieving this goal requires research into forwarding information efficiently from providers (wireless servers that detect the traffic event) through carriers ( $\mathrm{WiFi}$ access points moveable on lanes or near to traffic event) to consumers (vehicles that need such information). The consumers will likely be hundreds of meters behind the providers, and therefore will be out of direct radio range (i.e., the providers and consumers are not in the same neighborhood and thus are not neighbors).

The vehicles in the example above form an ad-hoc network, meaning that there is no pre-arranged network infrastructure and that the vehicles are not required to have access to the public Internet for communication. In addition, since vehicles are on mobility, so a mobile ad-hoc network (MANET) is created. In particular, MANETs that consist of vehicles as nodes, or vehicular ad-hoc networks (VANETs), face challenges which include high-speed mobility, constraints on vehicle lane movement, because of type of roadways and unpredictable driver behavior [2]. Vehicular Ad-Hoc Networks (VANETs) are a special kind of Mobile Ad-Hoc Network (MANET), where wireless-equipped (WiFi installed on road) vehicles form a network with no additional infrastructure.

WiFi[1],[15] also known as IEEE $802.11 \mathrm{~b}[16]$, is the preferred technology for wireless local area networking in both business and home environments. Even though it was designed primarily for private applications, $\mathrm{WiFi}$ is also being deployed in public places to create hotspots, where WiFi-capable users can obtain broadband Internet access. Using WiFi, user can connect to internet to get broadband access anywhere in home, office and public places etc., without using wires or cables.

\section{LITERATURE SURVEY}

I) The Travelpilot [3]: The Travelpilot is an effective, selfcontained, automotive navigation system that is use the U.S. and Europe. It provides needed assistance to individual drivers in finding their way over the road net. It can be easily expanded to serve fleet management applications. It provides the foundation for additional user functions like pathfinding, Yellow Pages, and traffic congestion display. It leads the way to improving drivers' use of their time and of transportation resources. The system uses dead-reckoning and map-matching for navigation.

2) Drawback of Dead Reckoning based Navigation system [7]: The dead-reckoning sensors in the original Navigator consisted of a magnetic compass (to measure vehicle heading) and wheel rotation sensors on the two undriven wheels (to measure distance traveled and turning information). Map matching was based on the assumption that if a vehicle traveled a reasonable distance at a reasonable speed it must be on a road. Probably the least reliable information in the Dead Reckoning + Map Matching (DR + $\mathrm{MM}$ ) system comes from the magnetic compass. It is not the compass itself that is at fault, but rather anomalies in the earth's magnetic field. These anomalies can be caused by 
steel reinforcements in elevated roads and overpasses, by steel enclosed bridges, by trolley tracks in the roadway, or by other metallic objects in, under, or near the road. Such magnetic anomalies can produce errors in measuring vehicle heading, and can result in the DR + MM system following the wrong road. To avoid the problem the Global Positioning System (GPS) based navigation system installed in vehicle.

3) TrafficRep [10]: The TrafficRep device connects to the invehicle navigation system, supplying it with current traffic conditions. From user's (driver) perspective. a user only interacts with the in-vehicle navigation system posing queries. TrafficRep device disseminates sensed traffic information to other vehicles. They act independently of other vehicles based on locally available information in order to decide what and when to disseminate. Each TrafficRep device maintains an estimate of the travel time on all the links. On receiving disseminated traffic reports, the vehicles update their travel time estimates. The widespread deployment of $\mathrm{WiFi}$ hotspots and wide area cellular networks opens up the exciting possibility of interoperability between these types of networks.

Interoperability allows a mobile device to dynamically use the multiple network interfaces available to it so as to maximize user satisfaction and system performance. The above figure 1.3 shows the interoperability of different network. The system under consideration consists of a set of Base Stations (BSs) belonging to a Packet Cellular Network that can provide wide area coverage to the Mobile Stations (MSs), and a set of Wi-Fi Access Points (APs) that provide high-speed connectivity to the MSs. Each MS is assumed to support only one wireless interface that can switch between the packet cellular and Wi-Fi modes of operation. The Wi-Fi APs are assumed to be inter-connected with one another, and with the BSs by means of either a wired backbone network, or by high bandwidth point-to-point wireless links. The BSs are connected by means of a high speed wired network.

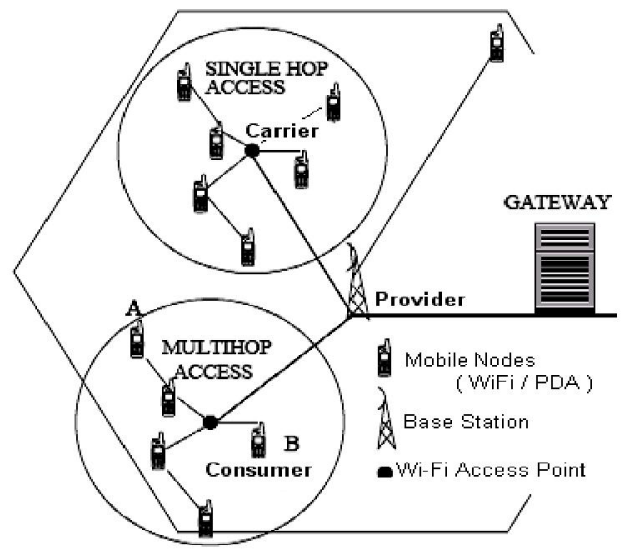

Fig. 2. VETRAC Inter-Operability Framework

A vehicle can utilize various communication types simultaneously. For example, a vehicle can send or listen to updates on traffic accidents through vehicle-vehicle communications as well as notify about driver or passengers health condition through vehicle-Internet communication.
The widespread deployment of WiFi hotspots and wide area cellular networks opens up the exciting possibility of interoperability between these types of networks. Interoperability allows a mobile device to dynamically use the multiple network interfaces available to it so as to maximize user satisfaction and system performance.

\section{B. Approach}

The application of inter-vehicular communication over a MANET raises several interesting issues in regard to data forwarding. One such issue is that an unpredictable traffic has both a limited area of relevance and a limited time of relevance. For example, carriers should not forward information about road closures after the road has re-opened. This requires carriers to not only forward received messages, but also analyze the semantics of the messages to first determine if they should be forwarded. As another example, carriers may be traveling to destinations hundreds of miles away from the traffic slowdown. It would be wasteful for these carriers to broadcast the notification of the traffic slowdown to all vehicles it encounters. VETRAC proposes to introduce relevance filtering at the carriers before forwarding event messages. Upon receiving an event message, the carrier will look at the location of the event and the expiration time of the event (e.g., lane re-opening time). If the carrier has exceeded a threshold distance away from the location or the expiration time of the event has passed, the carrier will discard the message. Otherwise, the carrier will add the message to its outgoing message queue.

Another issue to consider is network congestion. If all carriers are forwarding messages (and carriers in the same neighborhood would likely be forwarding the same messages), there will be many redundant messages on the network, hence carriers must filter messages to determine which ones are likely to be duplicated. To achieve this, VETRAC proposes to implement "redundant message" filtering at the carriers. If a carrier hears a message that matches one in its outgoing message queue, it will remove the message and add it to the end of its message queue. Additionally, the carrier could set a timer so that the message would not be sent until the timer expired, even if the message was at the front of the queue.

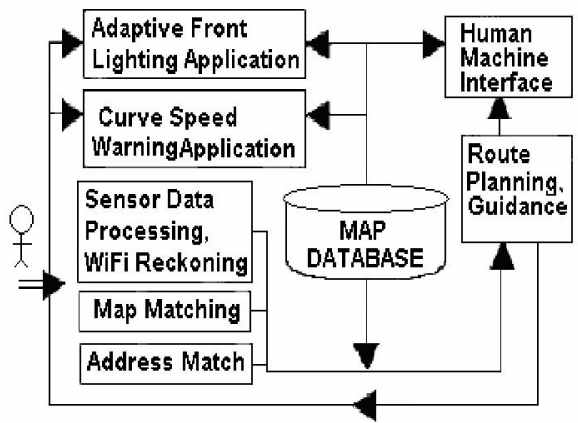

Fig. 3. Vehicle Tracking Setup

Nodes can use other techniques to further filter messages. For instance, we can assign a particular IP multicast group address to a physical area, and consumers can subscribe to 
multicast groups according to their current route. These consumers will only receive messages about those areas in which they are interested. Give all authors' names; use "et al." if there are six authors or more. Papers that have not been published, even if they have been submitted for publication, should be cited as "unpublished" [4]. Papers that have been accepted for publication should be cited as "in press" [5]. In a paper title, capitalize the first word and all other words except for conjunctions, prepositions less than seven letters, and prepositional phrases.

For papers published in translated journals, first give the English citation, then the original foreign-language citation [6].

\section{Vehicle Tracking ARCHTECTURE - VETRAC}

VETRAC system works on the principle identify of a vehicle, which is assigned as Mobile-IP address to a vehicle. The system tracks the vehicle information through WiFi access points, which are established at various locations in lane or parks or in large campus. The carrier is a navigation server that connects with multiple clients (vehicle) and is also responsible for the client's request data. The client control panel system (provider / consumer) running on client side helps the user for identifying current location, destination client location, and landmark, the distance to be traveled. Traffic intensity of each lane at an instant is notified though carrier access points.

\section{A. Functionality of VETRAC}

Fig-4 shows the functionality and architectural overview of Vehicle Tracking System (VETRAC), which consists of complex modules classified based on functionality. The core functionality of VETRAC focuses on vehicle tracking and controlling the lane (route) selection process.

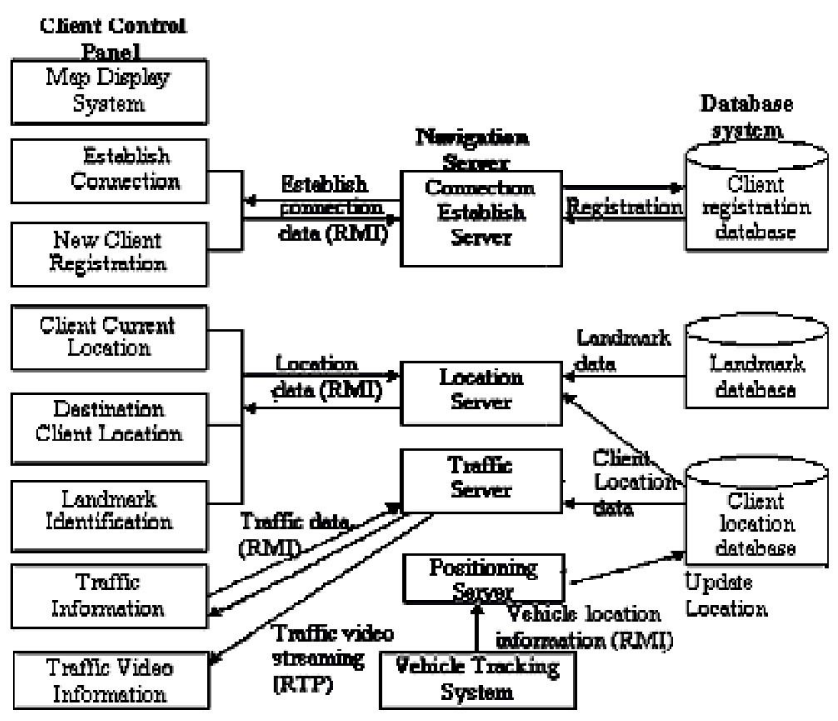

Fig. 4. VETRAC Functionality Frame-Work

1) Navigation Server: The core function of VETRAC System which mainly gathers handling request from client and tracking system. System establishes connection with client, updates the client location in database, gather the traffic information and controls streaming traffic video during conference process. The system has various modules

a. Connection establish server

b. Location Management Server and

c. Traffic server

2) Vehicle Tracking System: System connects with WiFi access point and tracking the client with in the access point range. The system detects the clients in its range and automatically pass the client current location to the navigation server.

3) Client Control Panel: The user can know about current location, destination client's location, landmark of the campus, traffic information in a campus, the traffic video information about junction in campus. The registered users can only access this navigation system. The system has various modules.

i. Establish connection

ii. New client registration

iii. Client current location

iv. Destination client's location

v. Traffic information

vi. Traffic video information

4) Display Map System: This system runs in the client device and it indicates the client's current location, destination client location, landmark identification and traffic information, in the map. It uses the image file for displaying map.

\section{IMPLEMENTATION}

We propose to launch a study of these issues in VANETs using Microsoft Windows-based personal digital assistants (PDAs) equipped with IEEE802.11 b/g (WiFi) receivers as the mobile nodes. We have chosen Microsoft Windows as the platform because Microsoft has partnered with several automobile manufacturers to develop a version of their operating system for use in vehicles [5]. We view any technology developed for such an inter-vehicular network to be easily deployable in vehicles that use Microsoft's automotive operating system.

A test bed has been designed for testing the prototype setup, which resembles our target application. To simulate a traffic build-up, a set of stationary providers have been prepared which could represent either emergency vehicles or vehicles that have detected a slowdown. To simulate oncoming traffic, vehicles in movement have been made to travel in differing speeds parallel to each other as well in opposite directions. Three WiFi access points have been used for the setup for a distance of 450 feet. These carriers will travel towards a set of consumers, which represent vehicles that have not yet approached the traffic buildup (and are not in the same neighborhood as the providers).

Multicast groups have been used for testing which require at least five PDAs or WiFi enabled laptops- one to function as the provider, two as the carriers, and two as the consumers. In total ten PDAs or WiFi enabled laptops as receivers have been used for setup. 


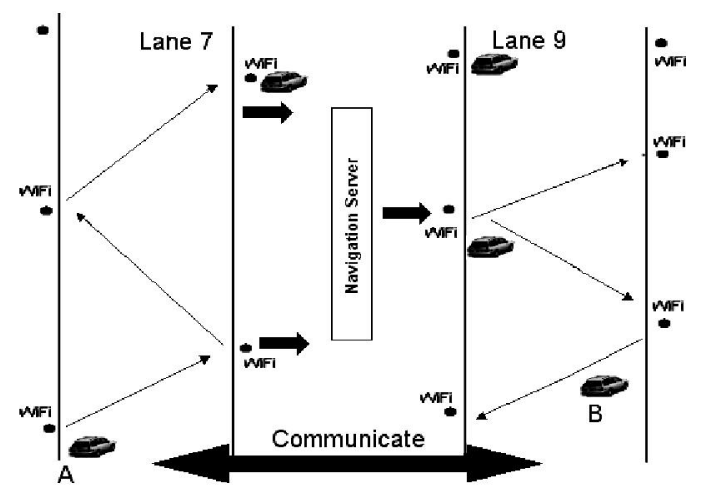

Fig. 5. Vehicle Traffic Navigate
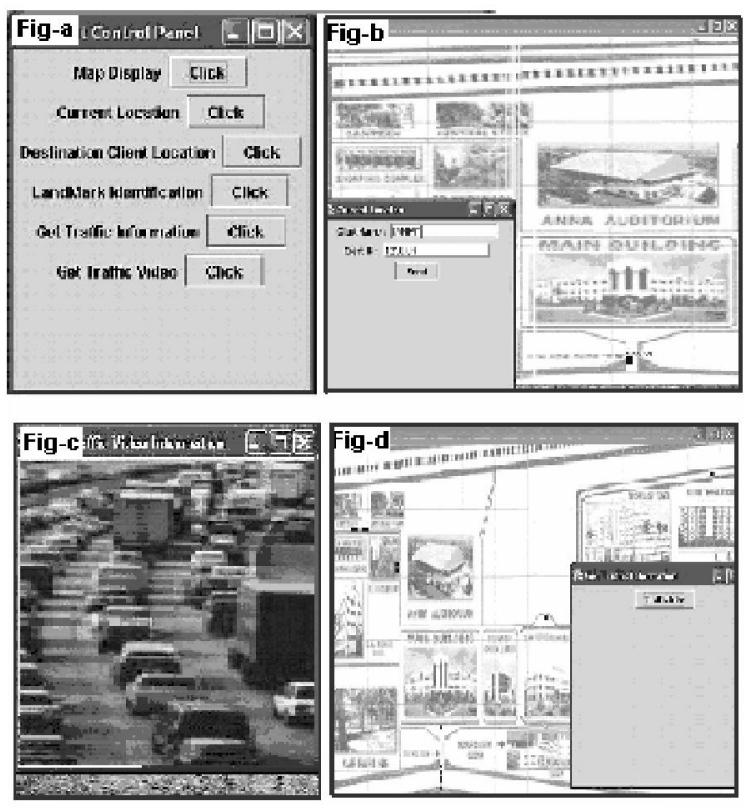

Fig. 6. VETRAC - Implementation and Results

Fig-6 shows VETRAC implementation inside an organizational campus, which encompasses over 25 acres of land and buildings. VETRAC helps to identify the buildings as well to navigate traffic. Fig-6a shows new registration of a client with VETRAC. Fig-6b shows VETRAC locating a building being searched by client. Fig- $6 \mathrm{c}$ displays the traffic intensity on a road requested by client. Fig- 6 d shows the map of requested place.

\section{CONClusion AND Future Work}

VETRAC system is a navigation system for large campus like University, Airport, Railway Stations, Organization. The main objective of this project is track vehicles, search and provide location information as well vehicle traffic information to drivers or users. This system contains rich, interactive graphical GUI form, which provides easy working for any new entrant into city or location or even a computer novice.
The advantage of this project is that it provides navigation function anywhere at anytime using WiFi wireless connection. Since GPS cannot be used to track vehicles inside a campus or a closed tunnel, WiFi has been employed. VETRAC is currently implemented for locations to be identified within range of 500 meters to 800 meters. Any user can easily access the system as well VETRAC system can be implemented in any vehicles.

The project is tested with various vehicles such as TATA Sumo cars and motor-cycles in the campus area somewhere the WiFi devices setup. Security of VETRAC has not been implemented in current setup, such that it can be taken up as future work. This project funded by NetResearchLabs for MTech - Computer Engineering students of VIT has been considered as one of the best project for the year 2005-06.

\section{REFERENCES}

[1] Bachir and Benslimane, "A Multicast Protocol in Ad-Hoc Networks: Inter-vehicle Geocast," IEEE Semiannual Vehicular Technology Conference, Spring 2003.

[2] Blum, J., A. Eskandarian, and L. Hoffman, "Challenges of Intervehicle Ad Hoc Networks," IEEE Transactions on Intelligent Transportation Systems, vol. 5, no. 4, December 2004.

[3] Briesemeister and Hommel, "Role-based Multicast in Highly Mobile But Sparsely Connected Ad Hoc Networks," ACM MobiHoc 2003.

[4] Ericsson, "Communication and Mobility by Cellular Advanced Radio," ComCar project web siet, ttp://www.comcar.de, December 2002.

[5] Goel, S.; Imielinski, T.; Ozbay, K., "Ascertaining viability of WiFi based vehicle-to-vehicle network for traffic information dissemination", IEEE Intelligent Transportation Systems, page(s): 1086 - 1091, Oct. 2004.

[6] Microsoft Corporation, "Windows Automotive Partners," http://www.microsoft.com/windows/embedded/

windowsautomotive/partners/default.mspx, last updated 2005.

[7] Montgomery, J., "A real-time traffic and weather reporting system for motorists", IEEE Consumer Communications and Networking Conference, page $580-581$, Jan. 2005.

[8] Sun et al. "GPS-based Message Broadcast for Adaptive InterVehicle Communications," IEEE Seminannual Vehicular Technology Conference, Fall 2000.

[9] Sweeney, L.E, "Comparative benefits of various automotive navigation and routing technologies", IEEE Position Location and Navigation Symposium, page 415 - 421, April 1996.

[10] Sweeney, L.E., Jr. Zavoli, W.B. Loughmiller, G., "Comparative performance of various automotive navigation technologies", IEEE Vehicle Navigation and Information Systems Conference, page(s): 437 - 440, Oct. 1993.

[11] Terry Costlow, "Wi-Fi Could Augment GPS", IEEE Distributed systems online, Vol. 5, Issue no. 9, Pages 1-2, Sep 2004.

[12] Ye, Tao, H.-Arno Jacobsen, and Randy Katz. "Mobile Awareness in a Wide Area Wireless Network of InfoStations," Proceedings of the 4th Annual ACM/IEEE International Conference on Mobile Computing and Networking, Dallas, Texas, 1998, pp. 109-120. 